Western University

Scholarship@Western

$11-30-2020$

\title{
Optimization of multi-electrode implant configurations and programming for the delivery of non-ablative electric fields in intratumoral modulation therapy.
}

Erin Iredale

Andrew Deweyert

Douglas A Hoover

Jeff Z Chen

Susanne Schmid

See next page for additional authors

Follow this and additional works at: https://ir.lib.uwo.ca/biophysicspub

Part of the Medical Biophysics Commons

Citation of this paper:

Iredale, Erin; Deweyert, Andrew; Hoover, Douglas A; Chen, Jeff Z; Schmid, Susanne; Hebb, Matthew O;

Peters, Terry M; and Wong, Eugene, "Optimization of multi-electrode implant configurations and programming for the delivery of non-ablative electric fields in intratumoral modulation therapy." (2020).

Medical Biophysics Publications. 530.

https://ir.lib.uwo.ca/biophysicspub/530 


\section{Authors}

Erin Iredale, Andrew Deweyert, Douglas A Hoover, Jeff Z Chen, Susanne Schmid, Matthew O Hebb, Terry M Peters, and Eugene Wong 
This is the peer reviewed version of the following article: Iredale E, Deweyert A, Hoover DA, Chen JZ, Schmid S, Hebb MO, Peters TM, Wong E. Optimization of multi-electrode implant configurations and programming for the delivery of non-ablative electric fields in intratumoral modulation therapy. Med Phys. 2020 Nov;47(11):5441-5454, which has been published in final form at https://doi.org/10.1002/mp.14496. This article may be used for non-commercial purposes in accordance with Wiley Terms and Conditions for Use of Self-Archived Versions.

\title{
Optimization of Multi-Electrode Implant Configurations and
}

\section{Programming for the Delivery of Non-Ablative Electric Fields in}

\section{Intratumoral Modulation Therapy}

\author{
Erin Iredale ${ }^{1}$, Andrew Deweyert ${ }^{2}$, Douglas A. Hoover ${ }^{1,4}$, Jeff Z. Chen ${ }^{1,4}$, Susanne Schmid ${ }^{2}$, \\ Matthew O. Hebb ${ }^{2,3}$, Terry M. Peters ${ }^{1,5}$, Eugene Wong ${ }^{1,6}$
}

${ }^{1}$ Department of Medical Biophysics, Schulich School of Medicine and Dentistry, Western University, London, ON, Canada.

${ }^{2}$ Department of Anatomy and Cell Biology, Schulich School of Medicine and Dentistry, Western University, London, ON, Canada.

${ }^{3}$ Department of Clinical Neurological Sciences, Schulich School of Medicine and Dentistry, Western University, London, ON, Canada.

${ }^{4}$ London Regional Cancer Program, London Health Sciences Centre, London, ON, Canada.

${ }^{5}$ Robarts Research Institute, Western University, London, ON, Canada.

${ }^{6}$ Department of Physics and Astronomy, Western University, London, ON, Canada. 
Corresponding Author E-mail: eiredale@uwo.ca

Running Title: Tumor Electric Field Optimization

Abstract - Purpose: Application of low intensity electric fields to interfere with tumor growth is being increasingly recognized as a promising new cancer treatment modality. Intratumoral modulation therapy (IMT) is a developing technology that uses multiple electrodes implanted within or adjacent tumor regions to deliver electric fields to treat cancer. In this study, the determination of optimal IMT parameters was cast as a mathematical optimization problem, and electrode configurations, programming, optimization, and maximum treatable tumor size were evaluated in the simplest and easiest to understand spherical tumor model. The establishment of electrode placement and programming rules to maximize electric field tumor coverage designed specifically for IMT is the first step in developing an effective IMT treatment planning system.

Methods: Finite element method electric field computer simulations for tumor models with 2 to 7 implanted electrodes were performed to quantify the electric field over time with various parameters, including number of electrodes (2 to 7 ), number of contacts per electrode ( 1 to 3 ), location within tumor volume, and input waveform with relative phase shift between 0 and $2 \pi$ radians. Homogeneous tissue specific conductivity and dielectric values were assigned to the spherical tumor and surrounding tissue volume. In order to achieve the goal of covering the tumor volume with a uniform threshold of $1 \mathrm{~V} / \mathrm{cm}$ electric field, a custom least square objective function was used to maximize the tumor volume covered by $1 \mathrm{~V} / \mathrm{cm}$ time averaged field, while maximizing the electric field in voxels receiving less than this threshold. An additional term in the objective function was investigated with a weighted tissue sparing term, to minimize the field to surrounding 
tissues. The positions of the electrodes were also optimized to maximize target coverage with the fewest number of electrodes. The complexity of this optimization problem including its nonconvexity, the presence of many local minima, and the computational load associated with these stochastic based optimizations led to the use of a custom pattern search algorithm. Optimization parameters were bounded between 0 and $2 \pi$ radians for phase shift, and anywhere within the tumor volume for location. The robustness of the pattern search method was then evaluated with 50 random initial parameter values.

Results: The optimization algorithm was successfully implemented, and for 2 to 4 electrodes, equally spaced relative phase shifts and electrodes placed equidistant from each other was optimal. For 5 electrodes, up to $2.5 \mathrm{~cm}$ diameter tumors with $2.0 \mathrm{~V}$, and $4.1 \mathrm{~cm}$ with $4.0 \mathrm{~V}$ could be treated with the optimal configuration of a centrally placed electrode and 4 surrounding electrodes. The use of 7 electrodes allow for $3.4 \mathrm{~cm}$ diameter coverage at $2.0 \mathrm{~V}$ and $5.5 \mathrm{~cm}$ at $4.0 \mathrm{~V}$. The evaluation of the optimization method using 50 random initial parameter values found the method to be robust in finding the optimal solution.

Conclusions: This study has established a robust optimization method for temporally optimizing electric field tumor coverage for IMT, with the adaptability to optimize a variety of parameters including geometrical and relative phase shift configurations.

Keywords - Optimization, Pattern Search Method, Electric Field, Tumor, Electrotherapy, Computer Simulation 


\section{Introduction}

There have been substantial recent advances in the application of electric fields to treat various forms of cancer ${ }^{1-12}$. Intratumoral modulation therapy (IMT) is a developing technology that uses implanted bioelectrodes to generate electric fields to control tumor growth ${ }^{10-12}$. By implanting electrodes directly within or adjacent the tumor volume, IMT has the potential to provide perpetual, titratable therapy for a variety of tumor types using a concealed, low maintenance delivery system. Preclinical studies in malignant brain tumors have demonstrated robust efficacy of IMT monotherapy at $200 \mathrm{kHz},+/-2 \mathrm{~V}$ stimulation through in vitro investigations of patient derived Glioblastoma Multiforme (GBM) cells $\left(65 \%\right.$ cell viability) ${ }^{11}$, and in vivo rodent models $(20 \% \text { reduction in tumor volume })^{11}$. While there is currently no observed impact on normal neurons or adverse neurological effects in the treated rodent cohort for low voltage $(2 \mathrm{~V})$, intermediate frequency $(200 \mathrm{kHz})$ electric fields ${ }^{11-12}$, surgical implantation of electrodes will pose the main safety concern with this treatment on human patients. A marked benefit of incorporating IMT within multi-modality treatment paradigms was observed through in vitro GBM models (cell viability reduction from $83 \%$ for temozolomide (TMZ) alone to $46 \%$ for combined IMT $+\mathrm{TMZ})^{11}$, and on patient derived diffuse intrinsic pontine glioma cells, where incorporating IMT with combined radiation and TMZ ( $44 \%$ vs. $60 \%$ cell viability) reduced cell viability to $20 \%{ }^{10}$. These studies were performed however using a non-optimized single electrode system with no phase shifting of input waveforms that was subsequently found to be limited by inadequate spatial coverage, with only $6.2 \%$ of the in vitro dish area covered by the desired $1 \mathrm{~V} / \mathrm{cm}^{10}$ and associated constraints on tumor control with $24 \%$ of an in vivo tumor receiving $1 \mathrm{~V} / \mathrm{cm}^{11}$. 
An extensive search of the published literature through databases including PubMed, Google Scholar and Scopus was completed using Boolean OR keywords such as tumor treating fields computer simulations and optimizations, implantable electrotherapeutic devices, deep brain stimulation, electroporation, electric field optimization, simulation-based optimization and nonconvex optimization. There are publications on optimizing electric field for treatment of tumors from external devices ${ }^{7-9,13,14-16}$, for deep brain stimulation with multiple contacts to steer the field to treat the intended millimeter sized target ${ }^{17}$, and for electroporation with multiple electrodes to cover tumor volumes with large field magnitude ${ }^{18-19}$. The present study is the first of its kind to extend and optimize the distribution of therapeutic-range IMT fields across tumor volumes using multiple implanted electrodes rather than a single stimulation source. Advantages of the present study include incorporating multiple electrodes with multiple contacts, phase shift of input waveforms, tissue sparing, algorithm robustness evaluation, and avoidance of local minima through a custom pattern search approach. Critical fundamental unknowns include the maximum tumor volumes attainable with multi-electrode IMT, configuration and placement of electrodes and selection of stimulation parameters to maintain a minimal stimulation voltage and number of electrodes, while still covering the tumor volume with the necessary electric field. Simulations in previous studies have found that single electrode stimulation only covers a small volume in both in vitro and in vivo experiments, and multiple electrodes would be required for necessary coverage $^{10,11} \cdot 1 \mathrm{~V} / \mathrm{cm}$ has been used as a threshold for in vitro ${ }^{4,5,10,11}$, in vivo $o^{4,5,11}$, and in Glioblastoma Multiforme clinical trials ${ }^{1-4,20}$. While it has been suggested that thresholds likely differ between tumor types ${ }^{10}$, this study will use a threshold of $1 \mathrm{~V} / \mathrm{cm}$ to demonstrate our optimization algorithm. However, the optimization method introduced in this study can use any desired field threshold. Simulations and optimizations have been established for an external 
device, but electric fields delivered using the external device require an accurate anisotropic conductivity whole brain map to determine the field to the tumor volume ${ }^{21-22}$. Conversely, electrodes placed internally within the tumor volume don't require the same accuracy of surrounding tissue conductivity anisotropies, as electric fields are not passing through layers of tissue with varying electrical properties.

As with early brachytherapy, a set of interstitial implant rules such as the Manchester system were established to serve as practical treatment planning guidelines for treating tumors of various sizes ${ }^{23}$. Both brachytherapy and IMT utilize multiple implanted sources (radiation vs. electric field) to deliver treatment, with large and steep "dose" gradients near the contact surface, so the experience gained from early brachytherapy treatment planning in terms of implant rules can be borrowed to initiate a set of rules for IMT. Providing analogous IMT rules in the present study for the number of electrodes required to cover various tumor sizes, the placement and programming of those electrodes, and starting points for patient specific treatment optimization will allow for clinical implementation of IMT.

Based on experience from external delivery of tumor treating fields ${ }^{7-9,13,14-16}$, deep brain stimulation optimizations ${ }^{17}$, and irreversible electroporation optimizations ${ }^{18-19}$, the incorporation of simulations and electric field optimizations ensure the desired field is being delivered to the tumor volume. The creation of methods to robustly optimize the electric field delivered to a tumor volume is a necessary step in the development of multi-electrode IMT. Stochastic optimization algorithms are designed to escape local minima by giving a finite probability to choose optimization parameters that steps away from the local minimum. Methods to evaluate the robustness of our algorithm have been considered in this study. We need to determine the configurations and stimulation parameters that result in the desired electric fields before 
proceeding to performing experiments in vitro and in vivo. In this study we propose the introduction of multiple stimulating electrodes with relative phase shifts between their respective stimulation waveforms, as a variable parameter to increase the electric field coverage over time. We demonstrate that the optimization of treatment parameters can be cast as a mathematical optimization problem, utilizing computer simulations to compute the electric field distribution over time. The goal of this study is to determine the optimal geometric electrode configurations and input waveform relative phase shifts, and to estimate the maximum treatable tumor volume for between 2 and 7 electrodes. To achieve this, we developed optimization methods that were utilized to form multi-electrode IMT configuration and programming rules for between 2 and 7 electrodes, to enable future applications to in vitro and in vivo preclinical models and patient specific human tumor scenarios.

\section{Materials and Methods}

\section{A. Electric Field Simulation}

The in silico tumor and electrode models were created in COMSOL Multiphysics (v5.4). Material properties were assigned to each geometrical entity in the model, including tumor, electrodes, and surrounding media. The optimization procedure in the present study does not depend on tumor site/location, though we require the electrical properties of the tumor and surrounding tissue. Since the electrical properties in the brain are most well known, these simulations were completed using human brain and tumor tissue as an example. A literature search of measured electrical properties of human brain and tumor tissues at $200 \mathrm{kHz}$ was performed, and standard relative dielectric $\varepsilon$ and conductivity $\sigma$ values for external tumor treating fields simulation 
and treatment planning were used $\mathrm{d}^{7-9,14-15,22}$, originally obtained from in vivo measurements on living tissues at comparable frequencies between 50 and $100 \mathrm{kHz}^{24-27}$. The relative dielectric and conductivity values for the tumor were 2000 and $0.24 \mathrm{~S} / \mathrm{m}$ respectively, and for the normal surrounding grey matter brain, 3000 and $0.25 \mathrm{~S} / \mathrm{m}^{7-9,14-15,22,24-27}$. White matter and grey matter can be incorporated as needed, since surrounding brain tissue depends on the tumor location. These conductivity and dielectric values were used to demonstrate the methods, but the pipeline allows users to input any necessary electrical properties. The example electrode material used here was platinum-iridium with a relative permittivity of $1^{28}$ and conductivity of $5.278 \times 10^{6} \mathrm{~S} / \mathrm{m}^{29}$. The electrodes were assigned a $0.8 \mathrm{~mm}$ radius to represent the upper range in deep brain stimulation electrode size $\mathrm{e}^{30-33}$, with variable contact height to allow for adequate depth coverage, location in polar coordinates $(r, \theta)$, and input voltage sinusoidal waveforms $(A \sin (2 \pi f t-\varphi))$, where $A$ is the amplitude, $f$ is the frequency of $200 \mathrm{kHz}, t$ is the time, and $\varphi$ is the phase shift. Non-ablative input voltage amplitudes of 2 and $4 \mathrm{~V}$ were applied in this study.

The tumor volume was given a variable diameter to allow for the computation of maximum tumor size versus number of electrodes. The total electrode contact height used was the tumor diameter $+2 \mathrm{~mm}$, to ensure adequate coverage at the poles, and for multiple contact electrodes, the spacing between contacts was $0.5 \mathrm{~mm}^{30-33}$. The height of exposed electrode contact is held constant between single, dual and three contact models. Starting with 2 single contact electrodes, the number of electrodes was increased to 3, 4, 5,6 and 7, and the number of contacts per electrode was also increased to 2 and 3 to further demonstrate our optimization algorithm in 3 dimensions (Fig. 1).

Using the AC/DC module in COMSOL Multiphysics, each electrode contact was given a separately programmed input voltage waveform terminal boundary. Any spacing between multiple 
contacts was insulated, satisfying $\mathbf{n} \cdot \mathbf{J}=0$ where $\mathbf{n}$ is the normal vector on the boundary, and $\mathbf{J}$ is the current density. Electrical insulation was also assumed on the outer boundary of the surrounding material. On internal boundaries between media with different electrical properties, continuity is maintained by the boundary condition $\mathbf{n}_{\mathbf{2}} \cdot\left(\mathbf{J}_{\mathbf{1}}-\mathbf{J}_{\mathbf{2}}\right)=0$, where subscripts 1 and 2 indicate the different media. A free tetrahedral mesh was created for the model, with varying sizes depending on the volume material. A time-dependent study was used to compute the electric field at 16 time points over half a period of the sinusoidal waveform. Using the electric currents user interface in COMSOL, a finite-element method was used to compute the electric field distribution on mesh points for our model. The governing equations for the finite element computation are Ohm's law (1), the equation of continuity (2), and Gauss' law (3):

$$
\begin{gathered}
\mathbf{J}(\mathbf{x}, t)=\sigma \mathbf{E}(\mathbf{x}, t) \\
\frac{\partial \rho(\mathbf{x}, t)}{\partial t}+\nabla \cdot \mathbf{J}(\mathbf{x}, t)=0 \\
\nabla \cdot \varepsilon \mathbf{E}(\mathbf{x}, t)=\rho(\mathbf{x}, t)
\end{gathered}
$$

where $\mathbf{J}(\mathbf{x}, t)$ is the current density as a function of location $\mathbf{x}$ and time $t, \sigma$ is the conductivity, $\mathbf{E}(\mathbf{x}, t)$ is the location and time dependent electric field, $\rho(\mathbf{x}, t)$ is the charge density and $\varepsilon$ is the dielectric constant. 


\section{B. Sensitivity Analysis}

In the present models of constant voltage stimulation, the impedance magnitude $|\mathbf{Z}|$ of the tissue,

$$
|\mathbf{Z}|=\left(\left(\frac{1}{R}\right)^{2}+(2 \pi f C)^{2}\right)^{-0.5}
$$

depends on resistance $R \propto 1 / \sigma$, frequency $f$ and capacitance $C \propto \varepsilon$. COMSOL was used to simulate the resistance and capacitance, and therefore impedance of the spherical tumor models. Depending on the number of electrodes, contacts and the geometrical configuration of each model, the resistance ranged from $100-400 \Omega$, and the capacitance was $\sim 10^{-10} \mathrm{~F}$. Based on the maximum simulated resistance of $400 \Omega$, and the capacitance of $10^{-10} \mathrm{~F}$, the impedance was calculated (Eqn. 4) for a range of frequencies from 0 to $4 \mathrm{MHz}$. The resistance term of the impedance $\left(R^{-2}\right)$ dominated the capacitance term $(2 \pi f C)^{2}$ up to $2 \mathrm{MHz}\left(R^{-1}>2 \pi f C\right)$. Above $2 \mathrm{MHz}$, the capacitance term dominated $\left(R^{-1}<2 \pi f C\right)$. For the frequency range in this study $(200 \mathrm{kHz})$, the resistance and therefore conductivity dominate the impedance. The sensitivity of the impedance to changes in frequency was examined from 0 to $4 \mathrm{MHz}$.

To examine the sensitivity of electric field distributions to changes in frequency, the electric field was simulated for the most complex 5 electrode geometry for a range of frequencies between 0 and $4 \mathrm{MHz}$. For each frequency, the resultant electric field matrices for 16 time points were averaged to obtain the temporal electric field magnitude $\bar{E}$. The relative difference $E_{\text {diff }}$ in the temporal average electric field magnitude $\bar{E}$ was calculated relative to the field map at the reference frequency $f_{\text {ref }}$ of $200 \mathrm{kHz}$. 


$$
E_{\text {diff }}=\frac{\left|\bar{E}(f)-\bar{E}\left(f_{\text {ref }}\right)\right|}{\bar{E}\left(f_{\text {ref }}\right)}
$$

Temporal average electric field maps were considered equivalent when $E_{\text {diff }}$ was less than $1 \%$. The sensitivity of electric field to changes in tumor conductivities were also analyzed (Eqn. 5), with $f$ and $f_{r e f}$ in (Eqn. 5) replaced with $\sigma$ and $\sigma_{r e f}$. Conductivities between 0.01 and $1 \mathrm{~S} / \mathrm{m}$ were analyzed. The relative dielectric constant sensitivity was analyzed with $f$ and $f_{\text {ref }}$ in (Eqn. 5) replaced with and $\varepsilon$ and $\varepsilon_{r e f}$ respectively for dielectrics ranging from 10 to 5000.

\section{Optimization Algorithm}

The optimization of the electric field coverage was coded in MATLAB and the COMSOL MATLAB Livelink used to connect our COMSOL model to our MATLAB code. We used the following least square objective function:

$$
F=\frac{1}{N_{s}} \sum_{j} \Theta\left(E_{\text {pres }}-\bar{E}_{j}\right)\left(\bar{E}_{j}-E_{\text {pres }}\right)^{2}
$$

where the sum is over the voxel $j, N_{s}$ is the number of voxels in the tumor volume, $\Theta$ is the Heaviside function, $E_{\text {pres }}$ is the prescription electric field threshold, and $\bar{E}_{j}$ is the time average electric field magnitude,

$$
\bar{E}_{j}=\frac{1}{N_{t}} \sum_{i} E_{i, j}
$$


where the sum is over time point $i$, and $N_{t}$ is the number of time points. This objective has been repurposed from radiotherapy ${ }^{34}$ to be used specifically for IMT delivery, and when minimized, optimizes the electric field coverage of the tumor over time. The Heaviside function was chosen to distinguish between covered voxels $\left(\bar{E}_{j} \geq E_{\text {pres }}\right)$ and uncovered voxels $\left(\bar{E}_{j}<E_{\text {pres }}\right)$, and the square difference term accounts for the contribution of uncovered voxels. The farther away a voxel is from the prescription field, the more it will affect the objective. Depending on tumor type, this prescription field can be changed to any necessary field magnitude. While externally delivered electric field optimizations typically maximize the average field to the tumor due to the inherently homogeneous nature of external delivery ${ }^{9,33}$, the nature of implanted electrodes, where the large electric fields and steep field gradients present near the electrode contact surface dominate the electric field average. While the average electric field to the tumor could be maximized using such objective functions, this could result in areas of the tumor not being covered by the desired field threshold in the case of implanted electrodes. Similar to brachytherapy, where a target volume is optimized to be covered by the prescription dose $\mathrm{e}^{23}$, we chose an objective function to allow optimization of the extent of tumor coverage with a more homogeneous electric field distribution to minimize cold $\operatorname{spots}^{34,35}$.

The algorithm includes an option to minimize the field delivered to regions outside the tumor volume, by adding a weighted term to the objective function,

$$
G=\frac{1}{N_{s}} \sum_{j} \Theta\left(E_{\text {pres }}-\bar{E}_{j}\right)\left(\bar{E}_{j}-E_{\text {pres }}\right)^{2}+w \frac{1}{N_{n}} \sum_{k}\left(\overline{E S}_{k}\right)^{2}
$$

where $w$ is the weighting factor, the second sum is over the normal tissue voxel $k, N_{n}$ is the number of voxels in the surrounding normal tissue, and $\overline{E S}_{k}$ is the time average electric field magnitude of 
normal tissue voxels. The weighting factor $w$ can be adjusted based on the importance of tissue sparing, and in this example a weighting factor of 0.1 was chosen based on trial optimizations $(w$ ranging from 0.05 to 1.00 ) to balance tumor coverage with the avoidance of critical tissue ${ }^{35}$. In addition to incorporating tissue sparing, weighted terms can be added to account for different tumor regions requiring different field thresholds depending on growth activity.

The optimization parameters used in this study were the location of each electrode $(r, \theta)$ (bounded to within tumor volume), and the phase shift $\varphi$ ( 0 to $2 \pi$ radians) of each electrode contacts input waveform. Models with between 2 and 7 electrodes with 1 to 3 contacts per electrode were investigated on spherical tumors up to $5.5 \mathrm{~cm}$ in diameter. The number of variables depends on the number of electrodes, and the number of contacts per electrode. In the present study, we held one electrode angle $\theta$ and one contact phase $\varphi$ constant at 0 as the reference. Therefore, if $n$ is the number of electrodes and $c$ is the number of contacts, a full parameter optimization contains $2 n+n c-2$ variables.

For each iteration of variables in the optimization algorithm, the electric field over time was computed in COMSOL, and the resultant field was linearly interpolated on a $100 \times 100 \times 100$ grid of equally spaced points covering the tumor volume. As our first step, we employed only electric field values within the tumor volume in the evaluation of the objective function.

To determine which optimization strategy would best fit this problem, we first determined whether our objective function was convex ${ }^{36}$. A function $F$ is convex if for all points in the domain $(\vec{x}, \vec{y}) \in \mathbb{R}^{n}$ and all $\lambda \in[0,1]:$

$$
F(\lambda \vec{x}+(1-\lambda) \vec{y}) \leq \lambda F(\vec{x})+(1-\lambda) F(\vec{y})
$$


By showing a single counter example to this inequality, we demonstrated that the problem is non-convex. This convexity test can be found in the supplementary materials.

In addition to this problem being non-convex for full location with phase optimizations, the computation of the gradient of our objective, which must be estimated by finite differences, is unreliable due to the mesh grid discretization of the electric field.

Due to the non-convexity of our problem and the unreliability of the gradient, we chose to customize the pattern search method, a gradient free, direct search optimization strategy for this study $^{37}$. This approach allows for a broader search of the parameter space, to help avoid falling into a local minimum. Each time the algorithm finds a better solution, the parameter step size is increased. Conversely, if no better point is found, the step size is decreased. The pattern search method was implemented using a custom MATLAB function that minimized our objective within certain bounds with a given starting point. Due to the uniqueness of our problem in determining IMT treatment parameters, a custom algorithm was developed. Within the algorithm, once the locations of the electrodes are selected for a given iteration, we made use of superposition of electric field from each electrode to optimize for its phase of the stimulation voltage. Such superposition of the electric field allows us to calculate the electric field once only and repeatedly use it during the voltage phase optimization. To avoid bias of user inputted starting points, parameter starting points were determined using $2 n$ random samples of parameter space, and to improve convergence rate, the objective function was evaluated for those $2 n$ samples, and the parameters resulting in the best objective were used as the starting point ${ }^{38-39}$. The overall pipeline of the optimization algorithm begins with the creation of the COMSOL model, with manual input of tumor volume (either simple geometry or any irregular shape) and tissue dielectric and conductivity properties, choice of optimization parameters and bounds (location, relative phase 
shift), choice of objective function (no tissue sparing or tissue sparing), and lastly, the pattern search optimization which evaluated the objective function based on the COMSOL simulations.

\section{Robustness of the Pattern Search Algorithm}

The robustness of our pattern search algorithm was evaluated for the 5 electrode 3 contact full phase optimization (14 variables relative to the top contact of electrode 1). Each of the 14 electrode contacts was assigned a random relative phase shift starting point for the optimization. The pattern search optimization was repeated for 50 more random starting points to evaluate the convergence of the pattern search algorithm to the optimal solution. The algorithm was considered converged to an optimal solution ${ }^{37-39}$ when the change in parameter value (step size) for an iteration was less than $1 \%$ of the range of the parameter (when phase shift parameter changes reached 0.063

radians $\left(\frac{2 \pi}{100}\right)$, and location changes were below $\left.0.3 \mathrm{~mm}\right)^{38-39}$. The starting parameters, optimal parameters, starting objective value, optimal objective value and number of iterations were all considered for each run of the algorithm.

\section{E. Spherical Tumor Optimal Configurations, Phase Shift and Maximum Treatable Tumor Size}

The maximum treatable tumor size for each number of electrodes was estimated by using the optimal geometric and phase configurations found by our algorithm. Since the optimal location within each configuration is dependent on the tumor size, the specific electrode locations were optimized for a range of tumor sizes. The electric field distributions for these optimal locations were then evaluated for the percentage of tumor volume covered by $95 \%$ of the prescription field $(1 \mathrm{~V} / \mathrm{cm})$. We defined the maximum treatable tumor size as the spherical diameter with at least $95 \%$ of the volume covered by a 95\% time average field "dose". This threshold was adopted from 
the minimum standard for radiotherapy treatment planning ${ }^{40-41}$. We began by optimizing the location and phase shifts of up to 7 electrode systems with single contact electrodes. Once we understood this, we optimized the configuration for electrodes with 2 and then 3 contacts.

\section{F. Tissue Sparing}

The optimization algorithm was applied to a tissue sparing example, where a $2.5 \mathrm{~cm}$ diameter spherical tumor was intersected by a $1.8 \mathrm{~cm}$ diameter cylindrical organ to be spared, creating a non-spherical target volume. The weighing factor in the objective $G$ was set to $w=0.1$ in this example. A full location and phase shift optimization of a 5 electrode single contact model was implemented in this investigation.

\section{Results}

\section{A. Sensitivity Analysis}

A sensitivity analysis was performed to investigate frequency, conductivity and dielectric constant effects on the electric field. There was less than $1 \%$ change in impedance over the range of $0-350 \mathrm{kHz}$ relative to the $200 \mathrm{kHz}$ impedance value. The relative difference in temporal average electric field maps was found to be less than $1 \%$ for frequencies ranging from 0 to $500 \mathrm{kHz}$ relative to the $200 \mathrm{kHz}$ reference field map. The relative difference in electric field maps was found to be less than $1 \%$ for conductivities ranging from 0.2 to $0.3 \mathrm{~S} / \mathrm{m}$ relative to the $0.24 \mathrm{~S} / \mathrm{m}$ reference field map, and dielectric constants between 500 and 4000 had equivalent field maps within 1\% difference relative to the 2000 dielectric reference field map. 


\section{B. Robustness of the Pattern Search Algorithm}

Our custom pattern search algorithm was robust in finding a global minimum of our optimization problem for the most complex case of full 14 variable phase optimization for the 5 electrode 3 contact model. For the 50 random starting parameter points, the optimization converged to a global optimum 45 times. The start and end objective values for all 50 runs are plotted in Fig. 2. Due to the large number of local minima and many equivalent global minima (due to symmetry) in our problem, and the uncertainty in the objective function value (due to the discretization of the electric field), the algorithm was considered successful in finding a global minima if the same objective value was reached to within a certain tolerance.

C. Spherical Tumor Optimal Configurations, Phase Shift and Maximum Treatable Tumor Size

\section{Single contact electrodes:}

Beginning with single contact electrodes and a spherical tumor model, we were able to use our optimization algorithm to find the configurations and relative phase shifts that resulted in the largest and most uniform electric field coverage over time. We then compared these optimal results to the field coverage when stimulating $2 \mathrm{~V}$ amplitude waveforms or ground electrodes were used, with no phase shifting.

The optimization of the 2 electrode model was completed in terms of the separation, and relative phase shift. The result was as expected, with maximally separated waveforms, with electrode 2 phase shifted $\pi$ radians from electrode 1 . We found that for the 2 electrode system, the maximum tumor diameter it can cover is $1.2 \mathrm{~cm}$, with electrodes placed at $\mathrm{r}=4 \mathrm{~mm}$ from the centre. The temporal average electric field map of the optimal configuration is found in Fig. 3, as 
well as the corresponding field animation over time. This result matched the expected solution of completely out of phase sine waves, to create the maximum electric potential difference (hence maximum electric field) between electrodes.

We found that for a 3 electrode system, the maximum tumor diameter it can treat is $1.7 \mathrm{~cm}$. Using maximum tumor size of $1.7 \mathrm{~cm}$ diameter, the optimal configuration found from our optimization was equally spaced input waveforms with phase shifts of $2 \pi / 3$ and $4 \pi / 3$ radians relative to electrode 1 . The optimal geometrical configuration was with electrodes placed equidistant from the centre, and each other. For this $1.7 \mathrm{~cm}$ diameter tumor volume, the optimal distance from the centre was $6 \mathrm{~mm}$. As can be seen in Fig. 3(a(ii))'s corresponding animation, these parameters lead to an electric field that rotates over time, encompassing the whole tumor volume, with no stagnant 'cold spots'. Since we are dealing with a spherically symmetric model, we expect that electrodes spaced equidistant from each other and from the centre of the sphere would be optimal. In addition, to create the most homogeneous electric field over time, maximally separated phase shifted input sine waves would lead to a symmetrically rotating electric field, which matched the optimization results.

For the 4 electrode model, the optimal configuration was all four electrodes placed equidistant from each other, with maximally separated input waveforms (phase shifts of $0.5 \pi, \pi$, $1.5 \pi$ relative to electrode 1 ) to create a symmetrically rotating electric field. We found that for a 4 electrode system, the maximum tumor diameter that can be treated is $2.1 \mathrm{~cm}$, for which the optimal distance from the centre for each electrode was $7.5 \mathrm{~mm}$.

Using a 5 electrode system, the maximum tumor diameter that can be treated is $2.5 \mathrm{~cm}$ with $2 \mathrm{~V}$ input waveforms. The optimal solution for this case was an electrode placed in the centre, with 4 equally spaced surrounding electrodes. The phase shifts relative to the centre electrode were 
$0.5 \pi, 1.5 \pi, 0.5 \pi, 1.5 \pi$ respectively. For a $2.5 \mathrm{~cm}$ diameter tumor, the optimal distance from the centre was $11 \mathrm{~mm}$. Field maps and animations of optimal solutions for both 4 and 5 electrode models can be found in Fig. 3(a). For the 5 electrode model, we compared the optimal results to the non-optimized configuration with no use of phase shifting (Fig. 3(b)). Since the electric field varied within the tumor volume, a cumulative electric field volume histogram $(\mathrm{EVH})^{6,9}$ was used to summarize the tumor coverage. We plotted the absolute tumor volume versus electric field strength in the EVH, indicating the minimum electric field strength that covers a certain volume of the tumor (Fig. 3(c)). For the 5 electrode configuration when no phase shifting is used, less than $20 \%$ of a $2.5 \mathrm{~cm}$ diameter tumor volume is covered by $1 \mathrm{~V} / \mathrm{cm}$, but for the optimized phase shifting case, $95 \%$ of the tumor volume is covered. This large tumor coverage increase can be observed when introducing phase shift to models with any number of electrodes.

Our methods were extended to investigate the optimal configurations and phase settings for 6 and 7 electrode models. For 6 electrodes, up to $2.94 \mathrm{~cm}$ diameter tumors can be covered using $2 \mathrm{~V}$ input waveforms with the optimal location of each electrode $(r, \theta)$ in $\mathrm{mm}$ and radians of $(0,0)$, $(13.75,0),(13,0.413 \pi),(11.5,0.850 \pi),(14.75,1.2 \pi),(11.5,1.588 \pi)$. The corresponding optimal phase shifts were $0,0.618 \pi, 1.460 \pi, 0.372 \pi, 0.938 \pi$, and $1.603 \pi$ radians. Next for 7 electrodes, up to $3.34 \mathrm{~cm}$ diameter tumors can be covered with a configuration of one central electrode, with 6 equally spaced surrounding electrodes at $13.2 \mathrm{~mm}$ from the centre. The optimal phase shifts of the surrounding electrodes alternate between $0.550 \pi$ and $1.436 \pi$, similar to the 5 electrode configuration. By using the optimal configurations found above, the maximum treatable tumor size (i.e., that with $95 \%$ coverage at $95 \%$ of the $1 \mathrm{~V} / \mathrm{cm}$ prescription field) can be determined for each number of electrodes with $2 \mathrm{~V}$ and $4 \mathrm{~V}$ input waveforms (Fig. 4). 


\section{Multi-Contact Electrodes:}

We further applied our optimization methods to multi-contact electrode models, with 2 or 3 contacts per electrode. The phase shift parameters of each additional contact were added to the optimization. Starting with a dual contact, 5 electrode model, the optimal configuration is equally spaced electrodes around a central electrode with phase shifts of the top contacts of $0,0.6 \pi, 1.4 \pi$, $0.6 \pi, 1.4 \pi$, and the bottom contacts $0,0.4 \pi, 1.6 \pi, 0.4 \pi, 1.6 \pi$, relative to the top centre electrode. When increasing the number of contacts to 2, our algorithm found an improvement in the objective function value when separating the phase of the top and bottom contact on an electrode. Compared to the optimal single contact configuration $(0.5 \pi, 1.5 \pi, 0.5 \pi, 1.5 \pi)$, the top contacts were shifted by $+0.1 \pi,-0.1 \pi,+0.1 \pi,-0.1 \pi$, and the bottom contacts were shifted by $-0.1 \pi,+0.1 \pi,-0.1 \pi$, $+0.1 \pi$ (Fig. 5).

Our algorithm was further applied to the 5 electrode, 3 contact model, where optimal phase shifts of contacts were $0,0.37 \pi, 1.63 \pi, 0.37 \pi, 1.63 \pi$ for top contacts, $0.10 \pi, 0.51 \pi, 1.58 \pi, 0.51 \pi$, $1.58 \pi$ for middle contacts and $0.40 \pi, 0.80 \pi, 1.35 \pi, 0.80 \pi, 1.35 \pi$ for bottom contacts, relative to the top centre contact. By adding a third contact to each electrode the objective was further improved, and each contact on an electrode had separated phase shift (Fig. 5). For single contact electrodes the electric field vectors rotate in cross sectional planes perpendicular to the electrode length. The addition of multiple contacts per electrode results in electric fields that vary in all three dimensions over time (Fig. 6). These additional contact phase parameters increase the flexibility of field shaping to cover any tumor shape and result in electric field vector rotation in three dimensions. 


\section{Tissue Sparing}

Our algorithm was applied to the tissue sparing example, where optimal electrode placements $(r, \theta)$ were found to be $(0,0),(11,0.37 \pi),(9,0.75 \pi),(9,1.25 \pi)$, and $(11,1.63 \pi)$. Optimal phase shifts were $0,0.10 \pi, 1.55 \pi, 0.45 \pi$, and $1.9 \pi$ radians. The input voltage required to ensure $95 \%$ of the tumor volume was covered by $95 \%$ of $1 \mathrm{~V} / \mathrm{cm}$ was $2.4 \mathrm{~V}$. The time average electric field map of the optimized configuration and corresponding EVH (Fig. 7) highlight the coverage of the tumor volume with $1 \mathrm{~V} / \mathrm{cm}$ electric field, while minimizing the field to the spared tissue.

\section{Discussion}

We have demonstrated that the problem of determining IMT treatment parameters can be cast as a mathematical optimization problem. Based on our results, it is clear that the use of relative phase shifts in the programming of electrode contacts allows for the custom design of location and time dependent electric vector fields that markedly enhance tumor coverage compared to nonphase shift settings (Fig. 3,6). By optimizing these parameters in addition to the electrode geometrical configuration and specific locations, we were able to maximize therapeutic field coverage for spherical tumors up to $5.5 \mathrm{~cm}$ diameter, and incorporate tissue sparing.

From the optimization results presented in Results section $C$. and Fig. 3 we have learned that for 2 to 4 electrodes, it is optimal to place electrodes equidistant from each other, and the centre, with equally spaced relative phase shifts. For 5 electrodes, with the increase in treatable tumor size, it becomes optimal to place an electrode in the centre, with 4 equally spaced surrounding electrodes. In general, the uniform distribution of electrodes within the tumor volume is the optimal configuration for all models we tested (Fig. 3). Based on this knowledge, 
optimizations could be improved by starting with electrodes uniformly distributed and optimizing the distance from the centre and phase configurations for specific tumors.

By increasing the number of electrodes used, the maximum treatable spherical tumor diameter is increased from $<1 \mathrm{~cm}$ for a single stimulating electrode to $1.2 \mathrm{~cm}, 1.7 \mathrm{~cm}, 2.1 \mathrm{~cm}, 2.5$ $\mathrm{cm}, 2.9 \mathrm{~cm}$ and $3.4 \mathrm{~cm}$ for 2, 3, 4, 5, 6 and 7 electrodes respectively for a $2 \mathrm{~V}$ amplitude input waveform (Fig. 4). By increasing the input voltage amplitude to $4 \mathrm{~V}$, the treatable tumor diameter is increased to $1.9 \mathrm{~cm}, 2.8 \mathrm{~cm}, 3.5 \mathrm{~cm}, 4.1 \mathrm{~cm}, 4.8 \mathrm{~cm}$ and $5.5 \mathrm{~cm}$ respectively (Fig. 4). The results presented for maximum tumor diameter versus number of electrodes (Fig. 4) were fit to the function $d=a \sqrt{n}+b$, as we expect that with additional electrodes, this relationship will approach an approximate square root dependence. The reason is as follows: Adding electrodes does not impact the ability to cover the longitudinal axis of the tumor (electrode length is always sufficient to cover this axis), but it does change the coverage of the cross-sectional plane of the tumor volume. Placement and phase shift optimization will spread the voltage gradients between electrodes spatially, maximizing the electric field coverage. As a result, after optimized for placement and phase shifts, each electrode will cover a similar area. Therefore, adding an electrode will increase the coverage area approximately linearly. Since the target coverage cross-sectional area varies with the square root of target radius (or diameter), we concluded that the target diameter coverage would vary with the square root of the number of electrodes. Up to the small range of 7 electrodes, the maximally covered tumor diameter almost appears to be linearly dependent on the number of electrodes.

In contrast to the additive effects of radioisotopes in brachytherapy, electric fields produced from multiple sources follow vector addition, leading to possible increased or decreased electric field strengths. The field cancellation feature can be exploited in tissue sparing situations. Hence, 
finding the locations and phase shifts to maintain sufficient field coverage within the tumor become increasingly complex and unintuitive with each additional electrode and the inclusion of critical structures. Such relationships will be further investigated in future studies. Incorporating tissue sparing into the objective function resulted in the tumor volume being covered by the desired $1 \mathrm{~V} / \mathrm{cm}$ electric field, while minimizing the field delivered to an intersecting sensitive structure (Fig. 7, Results $D$.). The configuration and phase setting rules presented in this study (Fig. 3, Results $C$.) were found to apply to frequencies up to $500 \mathrm{kHz}$, tumor conductivities between 0.2 and $0.3 \mathrm{~S} / \mathrm{m}$, and tumor dielectrics between 500 and 4000 (Results $A$.).

These results provide a set of rules as initial number, configurations and parameters for patient specific IMT treatment planning, where the size of tumor will be used to determine the number of electrodes required to cover that volume. If the patient tumor is approximately spherical, such as diffuse intrinsic pontine gliomas ${ }^{42}$, the configurations and phase settings outlined here for 2-7 electrodes could be used with distance from centre optimization. If the patient tumor shape is irregular, the configurations and settings could be used as a starting point for a full location and phase optimization. The optimization methods and results presented here will be validated by applying our methods to in vitro, in vivo and in human brain phantom experiments in the future.

This phase shifting approach to temporal electric field delivery maximizes the size of tumors that are possible to treat, while minimizing the number of electrodes. In addition, by incorporating multiple individually programmable contacts on each electrode, we were able to further improve the field coverage, and field shaping. Being able to control and optimize the distribution of the electric field produced, these methods could be further applied to irregularly shaped patient specific tumor contours in the future. Multiple contacts on each electrode increase the flexibility of our model, to adapt the electric fields to any tumor shape. Our objective function 
was also updated to incorporate tissue sparing and could be further modified to include tumor tissue inhomogeneity weighting terms, or adding a penalty term that would minimize the amount of power a configuration would require. In addition to increasing the magnitude of the temporal field coverage, incorporating relative phase shifts results in the electric field vector continuously changing direction. It has yet to be determined whether changing the direction of the electric field facilitates tumor cell death, but our method provides the capability to examine this hypothesis by designing experiments to investigate rotating field effects on the dielectrophoretic force hypothesis ${ }^{14,43-45}$, mitotic spindle microtubule interaction hypothesis ${ }^{14,43-45}$, and cell membrane effects $^{46}$. The application of our algorithm to design an in vitro experiment to investigate rotating fields can be found in the appendix. Such data could play a substantial role in informing future IMT advances.

Limitations of this study include the use of equal depth parallel electrodes. In reality, electrodes could have different insertion angles with different insertion depths. In future studies, this could be rectified by incorporating electrode angle and depth into the optimization simulations and by varying the input voltage amplitudes along the contacts of each electrode to compensate for differing separations along the electrode length. The tumor models investigated in this study utilized scalar conductivity, and while the sensitivity analysis showed anisotropies between 0.2 and $0.3 \mathrm{~S} / \mathrm{m}$ would have minimal effect $(<1 \%)$ on the electric field (Results $A$.), anisotropies outside this range could impact the distribution. Future studies will include patient-specific planning with anisotropies. Another limitation is that implanted electrodes result in a steep field gradient near the electrode surface, and while our optimization alleviated this and maximized the coverage, inhomogeneous fields remained as in brachytherapy. Similar to the comparison between external beam radiotherapy and brachytherapy, external electric field delivery could create more 
homogeneous fields, but the internal delivery has its benefits, such as reaching tumors in the deep brain and brainstem with more sparing capability for organs at risk, and providing a continuous treatment with no devices directly attached to the scalp which requires a shaved head. Heating mechanisms associated with implanted electrodes were also considered, to ensure that implanting electrodes at IMT voltages $(2 \mathrm{~V}-4 \mathrm{~V})$ and frequency $(200 \mathrm{kHz})$ would not result in adverse tissue heating or theoretical limitations to clinical implementation. At the low voltages proposed for IMT, Joule heating would theoretically contribute $<0.8^{\circ} \mathrm{C}$ to tissue directly adjacent the electrode surface, falling off with distance from the electrode ${ }^{47}$. At the intermediate frequency of $200 \mathrm{kHz}$, heating from dielectric losses are negligible, with the dielectric relaxation time of brain tissue remaining below the input waveform period, minimizing dielectric $\operatorname{losses}^{5,10-11,48}$. Limitations in the optimization algorithm pipeline include long computation times and manual input requirements which will need to be rectified before clinical implementation.

These baseline optimizations were performed on spherical tumor models in order to determine a set of rules for IMT treatment planning optimization. The optimization methods have been designed to allow users to input the required tissue electrical properties, import the tumor volume, choose the field threshold, and perform an optimization to find optimal electrode programming and placement. The incorporation of multiple separately programmable contacts on each electrode could allow for the optimization of irregularly shaped tumors to produce unique field coverage patterns. In addition, our algorithm can incorporate tissue sparing and organs at risk to minimize field exposure outside of the tumor. Overall, our optimization algorithm could be applied to any patient specific tumor model, for different electrode designs and optimization parameters. 


\section{Conclusions}

Our methods for temporally optimizing the electric field coverage with respect to relative phase shift programming, geometrical configurations, electrode and contact numbers for IMT are the first of their kind and have the adaptability to be able to incorporate a wider range of optimization parameters and tumor shapes/types in future investigations. This study has provided a baseline investigation and presentation of an optimization method for multi-electrode IMT, considering spherical tumor models. Current limitations include the use of parallel electrodes of equal depth, and the electric field inhomogeneities inherent to internal electrode placement. Overall, we have demonstrated the capability to optimize electrode placement and stimulation settings using methods developed specifically for IMT. These baseline tumor electrode configurations and phase setting rules are a critical first step in developing a patient specific treatment planning system for IMT.

\section{Appendix}

\section{Proposed In Vitro Experiment}

Our optimization algorithm was used to design a multi-electrode in vitro experiment to test the effects of electric field rotation. Four electrodes of $0.8 \mathrm{~mm}$ radius in a $1.75 \mathrm{~cm}$ radius in vitro dish were simulated in COMSOL. We first utilized our optimization algorithm to determine the 4 electrode geometry and input voltage amplitude of each electrode that results in a $1 \mathrm{~V} / \mathrm{cm}$ amplitude rotating electric field within a $0.5 \mathrm{~cm} \times 0.5 \mathrm{~cm}$ viewing window. The updated objective 
function $F=\frac{1}{N_{s}} \sum_{j}\left(\bar{E}_{j}-E_{\text {pres }}\right)^{2}$ is the square difference of the average temporal electric field and the prescription $1 \mathrm{~V} / \mathrm{cm}$ field.

The closer the majority of voxels are to $1 \mathrm{~V} / \mathrm{cm}$, the lower the objective value. Since we are looking at a 4 electrode rotating model, phase shifts for this optimization were set to $0,0.5 \pi$, $\pi, 1.5 \pi$ radians, and electrodes were equally spaced. The optimization algorithm was used to find the optimal input voltage and distance from the centre of the electrodes, to ensure a continuous 1 $\mathrm{V} / \mathrm{cm}$ is applied in the viewing window. Next, our optimization algorithm was used to determine the placement of electrodes and amplitude of input square waves that result in a $1 \mathrm{~V} / \mathrm{cm}$ nonrotating electric field.

We found that equally spaced electrodes placed $1.5 \mathrm{~cm}$ from the centre with input voltage amplitudes of $5.17 \mathrm{~V}$ led to a continuously rotating electric field of $1.00 \pm 0.01 \mathrm{~V} / \mathrm{cm}$ temporal average within the viewing window. The non-rotating electric field optimization resulted in all 4 electrodes placed $1.27 \mathrm{~cm}$ from the centre at angles of \pm 0.488 and \pm 2.663 radians with alternating input voltages of $5.17 \mathrm{~V}$ and $0 \mathrm{~V}$. This led to a time average electric field of $1.00 \pm 0.03 \mathrm{~V} / \mathrm{cm}$ (Fig. 8).

\section{Acknowledgements}

This work was supported in part by NSERC CGS-M (E.I.), NSERC Discovery Grant, Cancer Research Society, and Western Innovation Fund. 


\section{Conflicts of Interest}

M.O.H. and S.S. are inventors on the following patent applications that are assigned to “London Health Sciences Centre Research Inc.” (the applicant): PCT/CA2016/050556 entitled "Intratumoral Modulation Therapy" and corresponding national phase applications in Canada, United States, Europe and China. E.W., M.O.H., T.P., and S.S. are inventors on the following patent application that is assigned to "London Health Sciences Centre Research Inc." (the applicant): US provisional Serial No. 62/962,553 entitled "Planning and delivery of dynamically oriented electric field for biomedical applications". The authors have no other competing interests.

\section{References}

1. Hottinger AF, Pacheco P, Stupp R. Tumor treating fields: A novel treatment modality and its use in brain tumors. Neuro Oncol. 2016;18(10):1338-49. doi:10.1093/neuonc/now182.

2. Swanson KD, Lok E, Wong ET. An Overview of Alternating Electric Fields Therapy (NovoTTF Therapy) for the Treatment of Malignant Glioma. Curr Neurol Neurosci Rep. 2016;16(1):8. doi:10.1007/s1 1910-015-0606-5.

3. Stupp R, Taillibert S, Kanner A, et al. Effect of tumor-treating fields plus maintenance temozolomide vs maintenance temozolomide alone on survival in patients with glioblastoma a randomized clinical trial. JAMA - $J$ Am Med Assoc. 2017;318(23):2306-2316. doi:10.1001/jama.2017.18718.

4. Kirson ED, Dbaly V, Tovarys F, et al. Alternating electric fields arrest cell proliferation in animal tumor models and human brain tumors. Proc Natl Acad Sci U S A. 2007;104(24):10152-10157. doi:10.1073/pnas.0702916104. 
5. Kirson ED, Gurvich Z, Schneiderman R, et al. Disruption of cancer cell replication by alternating electric fields. Cancer Res. 2004;64(9):3288-3295. doi:10.1158/0008-5472.can-04-0083.

6. Lok E, San P, Hua V, Phung M, Wong ET. Analysis of physical characteristics of Tumor Treating Fields for human glioblastoma. Cancer Med. 2017;6(6):1286-1300. doi:10.1002/cam4.1095.

7. Wenger C, Salvador R, Basser PJ, Miranda PC. Improving Tumor Treating Fields Treatment Efficacy in Patients with Glioblastoma Using Personalized Array Layouts. Int J Radiat Oncol Biol Phys. 2016;94(5):1137-43. doi:10.1016/j.ijrobp.2015.11.042.

8. Wenger C, Salvador R, Basser PJ, Miranda PC. The electric field distribution in the brain during TTFields therapy and its dependence on tissue dielectric properties and anatomy: A computational study. Phys Med Biol. 2015;60(18):7339-57. doi:10.1088/0031-9155/60/18/7339.

9. Korshoej AR, Saturnino GB, Rasmussen LK, Von Oettingen G, Sørensen JCH, Thielscher A. Enhancing predicted efficacy of tumor treating fields therapy of glioblastoma using targeted surgical craniectomy: A computer modeling study. PLoS One. 2016;11(10):e0164051. doi:10.1371/journal.pone.0164051.

10. Deweyert A, Iredale E, Xu H, Wong E, Schmid S, Hebb MO. Diffuse intrinsic pontine glioma cells are vulnerable to low intensity electric fields delivered by intratumoral modulation therapy. $J$ Neurooncol. 2019;143:49-56. doi:10.1007/s11060-019-03145-8.

11. Di Sebastiano AR, Deweyert A, Benoit S, et al. Preclinical outcomes of Intratumoral Modulation Therapy for glioblastoma. Sci Rep. 2018;8:7301. doi:10.1038/s41598-018-25639-7.

12. Xu H, Bihari F, Whitehead S, Wong E, Schmid S, Hebb MO. In vitro validation of intratumoral modulation therapy for glioblastoma. Anticancer Res. 2016;36(1):71-80.

13. Naveh A, Bomzon Z, Farber O, et al. Transducer array configuration optimization for treatment of pancreatic cancer using Tumor Treating Fields (TTFields). Cancer Res. 2018;78(13): 2. doi:10.1158/1538-7445.AM2018-3204. 
14. Wenger C, Miranda PC, Salvador R, et al. A Review on Tumor-Treating Fields (TTFields): Clinical Implications Inferred From Computational Modeling. IEEE T Bio-Med Eng. 2018;11:195-207. doi:10.1109/RBME.2017.2765282.

15. Miranda PC, Mekonnen A, Salvador R, Basser PJ. Predicting the electric field distribution in the brain for the treatment of glioblastoma. Phys Med Biol. 2014;59(15):4137-47. doi:10.1088/0031$9155 / 59 / 15 / 4137$.

16. Sung J, Seo J, Jo Y, Yoon M, Hwang SG, Kim EH. Development of a Method for Improving the Electric Field Distribution in Patients Undergoing Tumor-Treating Fields Therapy. J Korean Phys Soc. 2018;73:1577-1583. doi:10.3938/jkps.73.1577.

17. Cubo R, Fahlström M, Jiltsova E, Andersson H, Medvedev A. Calculating deep brain stimulation amplitudes and power consumption by constrained optimization. $J$ Neural Eng. 2019;16(1):016020. doi:10.1088/1741-2552/aaeeb7.

18. Adeyanju OO, Al-Angari HM, Sahakian AV. The optimization of needle electrode number and placement for irreversible electroporation of hepatocellular carcinoma. Radiol Oncol. 2012;46(2):126-135. doi:10.2478/v10019-012-0026-y.

19. Garcia PA, Kos B, Rossmeisl Jr JH, Pavilha D, Miklavčič D, Davalos RV. Predictive therapeutic planning for irreversible electroporation treatment of spontaneous malignant glioma. Med Phys. 2017;44(9):4968-4980. doi:10.1002/mp.12401.

20. Ballo MT, Urman N, Lavy-Shahaf G, Grewal J, Bomzon Z, Toms S. Correlation of Tumor Treating Fields Dosimetry to Survival Outcomes in Newly Diagnosed Glioblastoma: A Large-Scale Numerical Simulation-Based Analysis of Data from the Phase 3 EF-14 Randomized Trial. Int J Radiation Oncol Biol Phys. 2019;104(5):1106-1113. doi:10.1016/j.ijrobp.2019.04.008.

21. Korshoej AR, Sørensen JCH, von Oettingen GB, Poulsen FR, Thielscher A. Optimization of tumor treating fields using singular value decomposition and minimization of field anisotropy. Phys Med Biol. 2019;64(4): 9. doi:10.1088/1361-6560/aafe54. 
22. Korshoej AR, Hansen FL, Thielscher A, von Oettingen GB, Sørensen JCH. Impact of tumor position, conductivity distribution and tissue homogeneity on the distribution of tumor treating fields in a human brain: A computer modeling study. PloS One. 2017;12(6):e0179214. doi:10.1371/journal.pone.0179214.

23. Dutreix A. Can we compare systems for interstitial therapy? Radiother Oncol. 1988;13(2):127-135. doi:10.1016/0167-8140(88)90033-3.

24. Gabriel C, Peyman A, Grant EH. Electrical conductivity of tissue at frequencies below $1 \mathrm{MHz}$. Phys Med Biol. 2009;54:4863-4878. doi:10.1088/0031-9155/54/16/002.

25. Latikka J, Kuurne T, Eskola H. Conductivity of living intracranial tissues. Phys Med Biol. 2001;46:1611-1616. doi:10.1088/0031-9155/46/6/302.

26. Stoy RD, Foster KR, Schwan HP. Dielectric properties of mammalian tissues from 0.1 to $100 \mathrm{MHz}$ : a summary of recent data. Phys Med Biol. 1982;27(4):501-513. doi:10.1088/0031-9155/27/4/002.

27. Latikka J, Eskola H. The Resistivity of Human Brain Tumours In Vivo. Ann Biomed Eng. 2019;47(3):706-713. doi:10.1007/s10439-018-02189-7.

28. Lourtioz JM, Benisty H, Berger V, Gerard JM, Maystre D, Tchelnokov A. Photonic Crystals: Towards Nanoscale Photonic Devices. Springer. 2008; pp. 122-123.

29. CSNDT Conductivity of Materials. Eddy Current Testing Manual on Eddy Current Method, NonDestructive Testing Magazine Sept/Oct 1955, Cosgrove Article.

30. Amon A, Alesch F. Systems for deep brain stimulation: review of technical features. J Neural Transm (Vienna). 2017;124(9):1083-1091. doi:10.1007/s00702-017-1751-6.

31. Butson C, McIntyre C. Role of electrode design on the volume of tissue activated during deep brain stimulation. J Neural Eng. 2006;3(1):1-8. doi:10.1088/1741-2560/3/1/001.

32. Alonso F, Latorre MA, Goransson N, Zsigmond P, Wardell K. Investigation into Deep Brain Stimulation Lead Designs: A Patient-Specific Simulation Study. Brain Sci. 2016;6(3). doi:10.3390/brainsci6030039. 
33. Anderson DN, Osting B, Vorwerk J, Dorval AD, Butson CR. Optimized programming algorithm for cylindrical and directional deep brain stimulation electrodes. J Neural Eng. 2018;15(2):026005. doi:10.1088/1741-2552/aaa14b.

34. Wieser HP, Cisternas E, Wahl N, et al. Development of the Open-Source Dose Calculation and Optimization Toolkit matRad. Med Phys. 2017;44(6):2556-2568. doi:10.1002/mp.12251.

35. Orton CG, Bortfeld TR, Niemierko A, Unkelbach J. The role of medical physicists and the AAPM in the development of treatment planning and optimization. Med Phys. 2008;35(11):4911-4923. doi:10.1118/1.2990777.

36. Boyd SP, Vandenberghe L. Convex Optimization. Cambridge University Press. 2004.

37. Amaran S, Sahinidis NV, Sharda B, Bury SJ. Simulation optimization: a review of algorithms and applications. Ann Oper Res. 2016;240(1):351-380. doi:10.1007/s10479-015-2019-x.

38. Nocedal J, Wright SJ. Numerical Optimization. New York: Springer; 2006.

39. Lange K. Optimization. New York: Springer; 2013.

40. International Commission on Radiation Units and Measurements. ICRU Report 62: Prescribing, recording and reporting photon beam therapy (Supplement to ICRU report 50). 1999.

41. Murthy KK, Shukeili KA, Kumar SS, Davis CA, Chandran RR, Namrata S. Evaluation of dose coverage to target volume and normal tissue sparing in the adjuvant radiotherapy of gastric cancers: 3D-CRT compared with dynamic IMRT. Biomed Imaging Interv J. 2010;6(3):e29. doi:10.2349/biij.6.3.e29.

42. Matthew RK, Rutka JT. Diffuse Intrinsic Pontine Glioma: Clinical Features, Molecular Genetics, and Novel Targeted Therapeutics. J Korean Neurosurg Soc. 2018;61(3):343-351. doi:10.3340/jkns.2018.0008.

43. Wenger C, Giladi M, Bomzon Z, Salvador R, Basser PJ, Miranda PC. Modeling Tumor Treating Fields (TTFields) application in single cells during metaphase and telophase. Conf Proc IEEE Eng Med and Biol Soc. 2015;6892-5. doi:10.1109/EMBC.2015.7319977. 
44. Gera N, Yang A, Holtzman TS, Lee SX, Wong ET, Swanson KD. Tumor treating fields perturb the localization of septins and cause aberrant mitotic exit. PLoS One. 2015;10(5):e0125269. doi:10.1371/journal.pone.0125269.

45. Berkelmann L, Bader A, Meshksar S, et al. Tumor-treating fields (TTFields): Investigations on the mechanism of action by electromagnetic exposure of cells in telophase/cytokinesis. Sci Rep. 2019;9:7362. doi:10.1038/s41598-019-43621-9.

46. Chang E, Patel CB, Pohling C, et al. Tumor treating fields increases membrane permeability in glioblastoma cells. Cell Death Discov. 2018;4:113. doi:10.1038/s41420-018-0130-x.

47. Elwassif MM, Kong Q, Vazquez M, Bikson M. Bio-heat transfer model of deep brain stimulationinduced temperature changes. J Neural Eng. 2006;3(4)306-315. doi:10.1088/1741-2560/3/4/008.

48. Lok E, Sajo E. Fundamental Physics of Tumor Treating Fields. In: Wong E. Alternating Electric Fields Therapy in Oncology. Springer. 2016;15-27. 


\section{Figures}

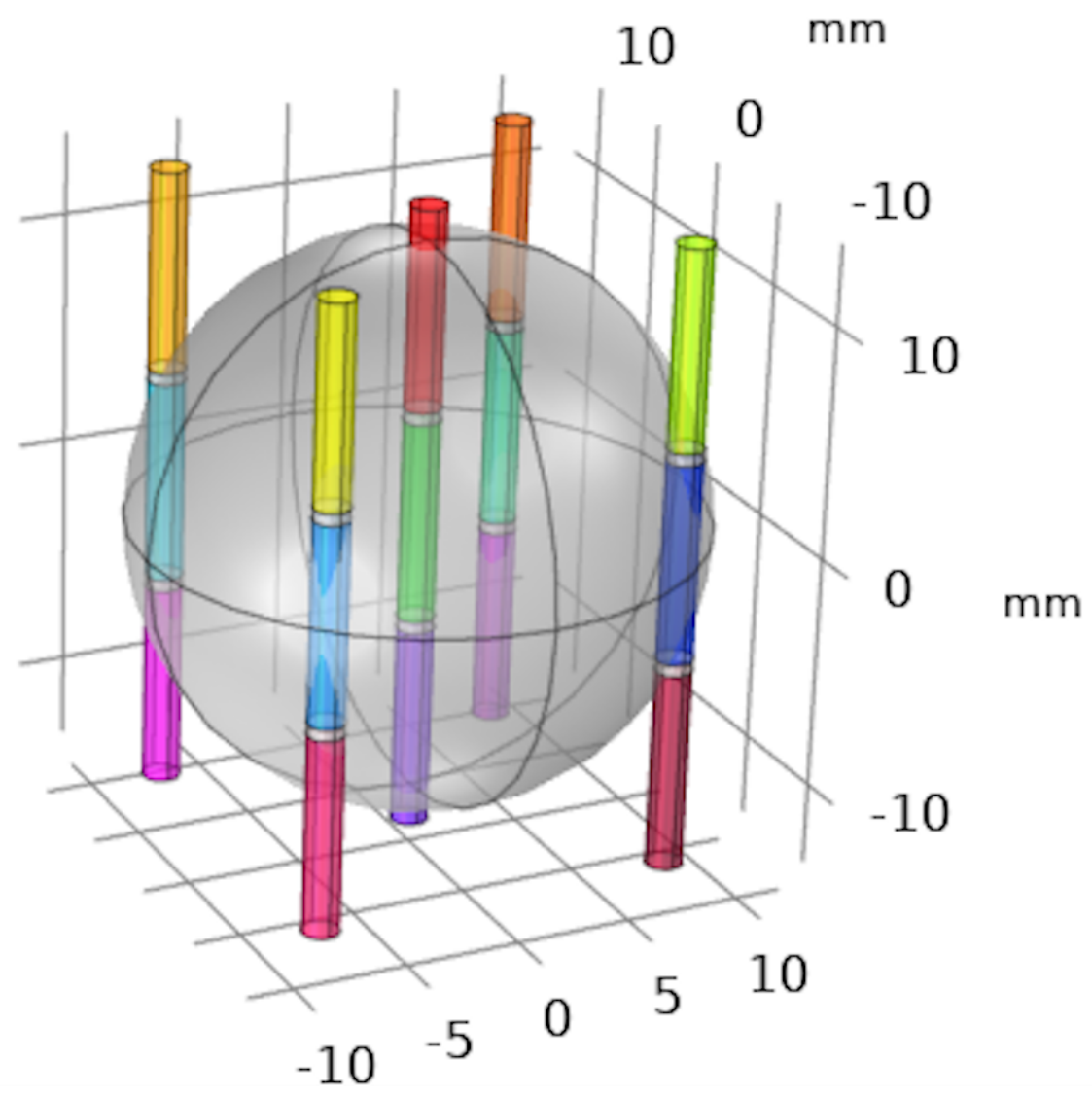

Fig. 1. Geometry of a 5 electrode, 3 contact tumor model (grey) created in COMSOL. Total of 15 contacts, each with separately programmable input waveforms highlighted by different colours. The tumor is surrounded by normal tissue. 


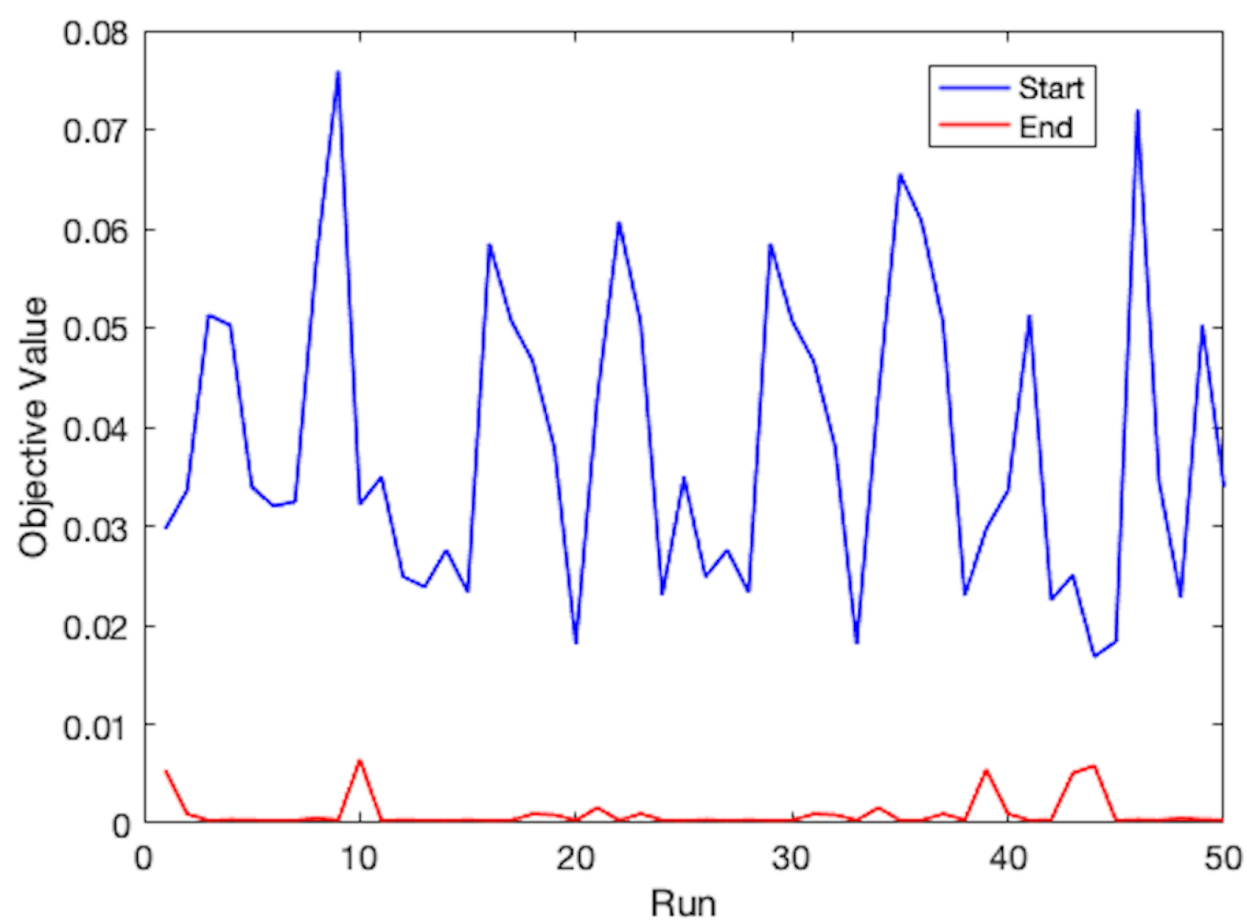

Fig. 2. Objective function value for starting random parameters and final minimized objective value for 50 runs with different random starting parameters. 

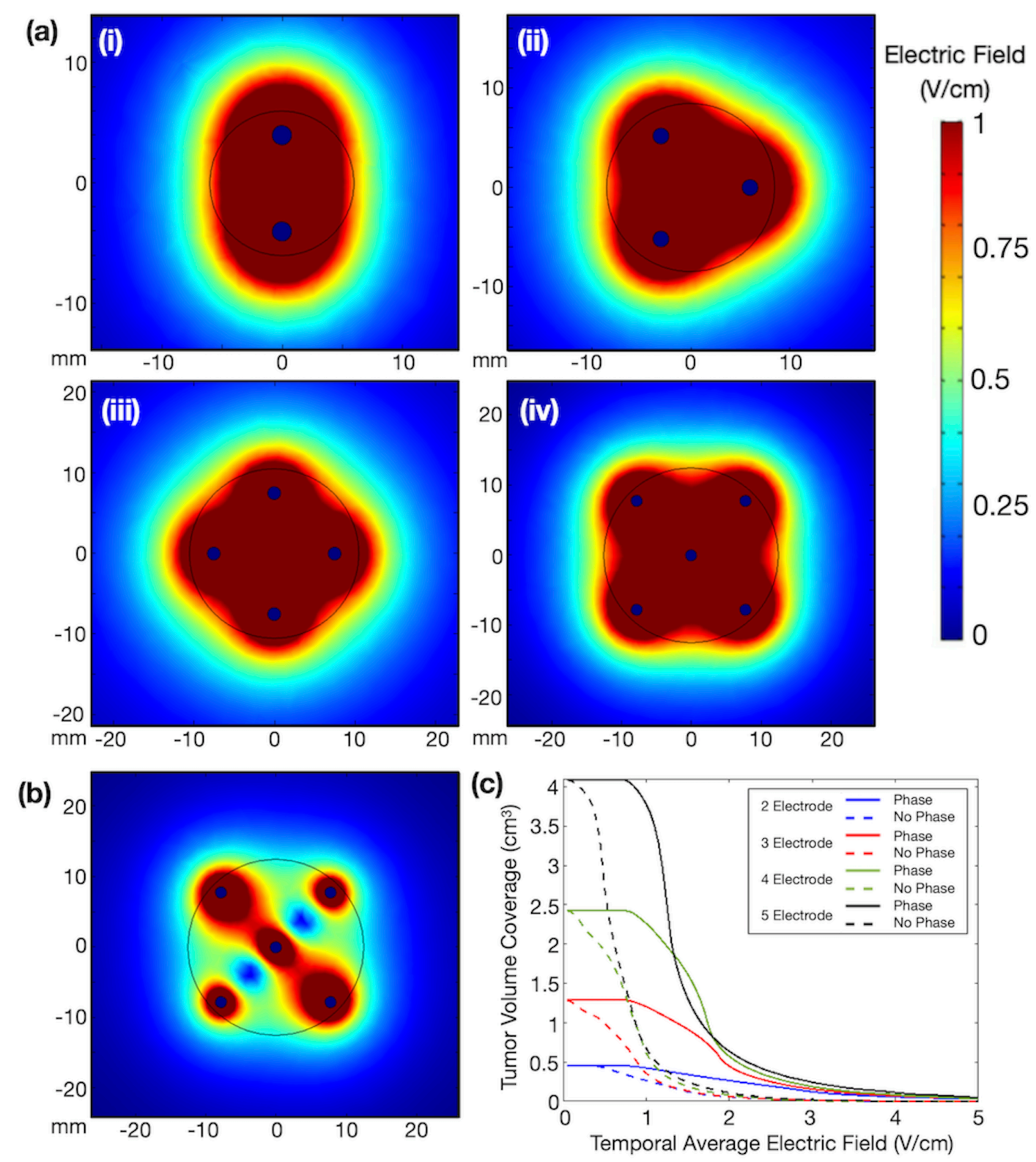

Fig. 3. Average temporal electric field maps and corresponding animations of field coverage over time for (a) 2, 3, 4, and 5 optimally placed electrodes in cross sections of corresponding maximum tumor volumes (black circle). (b) 5 electrode configuration with no phase shifting (centre, upper left, bottom right: $2 \mathrm{~V}$ amplitude stimulating electrodes, upper right and bottom left: $0 \mathrm{~V}$ ground electrodes). 2, 3, and 4 electrode figures with no phase shifting can be found in the supplementary materials (Fig. S-1). (c) Electric field volume histogram of the tumor volume $\left(\mathrm{cm}^{3}\right)$ receiving at least a certain temporal average electric field value $(\mathrm{V} / \mathrm{cm})$ for both optimized phase shift and no phase shift cases. 


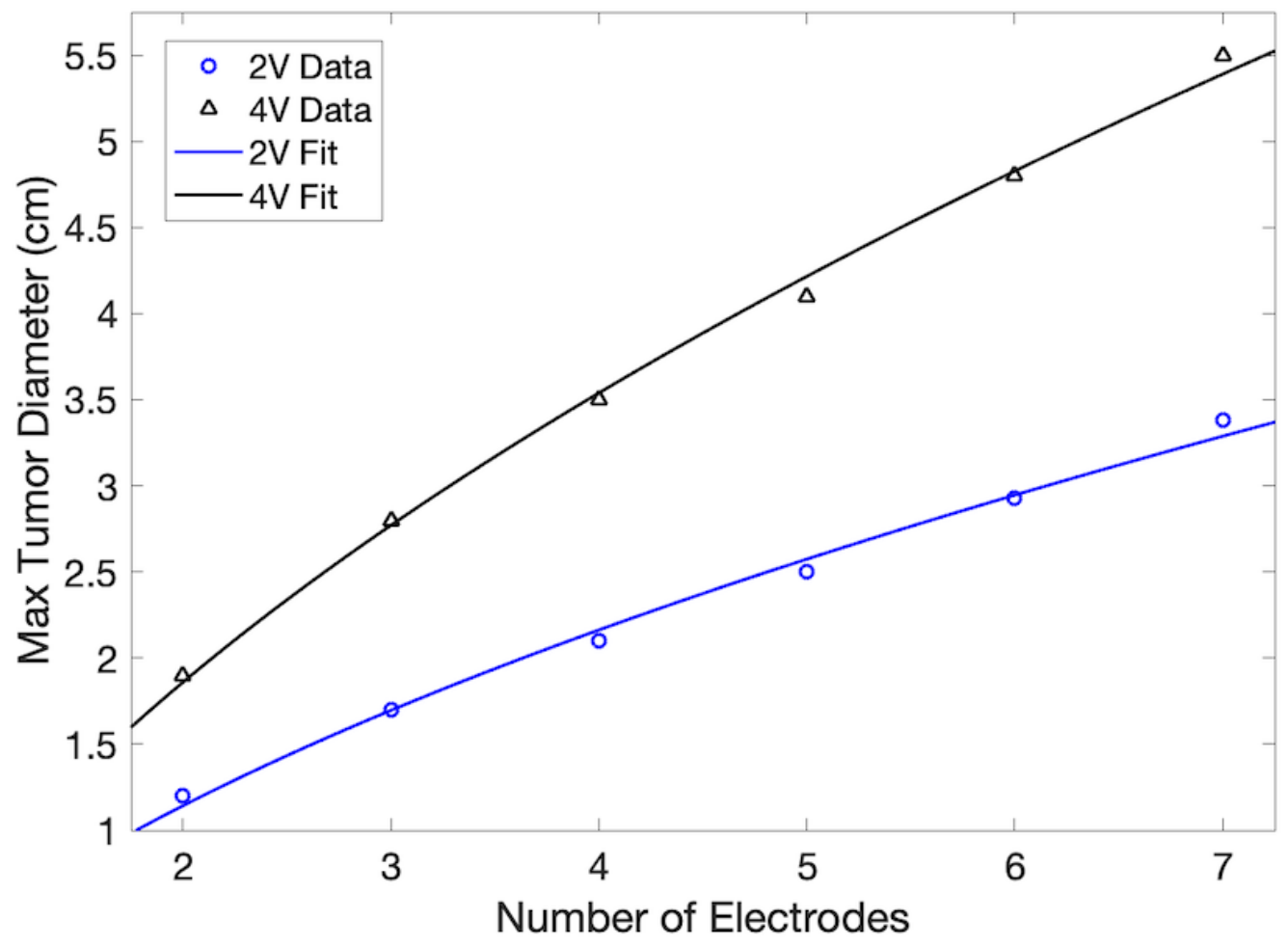

Fig. 4. Maximum treatable tumor diameter ( $95 \%$ coverage of $95 \%$ of prescription $1 \mathrm{~V} / \mathrm{cm}$ ) for 2, 3, 4, 5, 6 and 7 single contact electrodes at both $2 \mathrm{~V}$ and $4 \mathrm{~V}$ input sine wave amplitudes. The data was fit to a function of $d=a \sqrt{n}+b$, where $d$ is the tumor diameter, $n$ is the number of electrodes, and $a$ and $b$ are fit parameters. With 7 electrodes, at $4 \mathrm{~V}$, with optimal location and phase shift, tumors up to $5.5 \mathrm{~cm}$ diameter could be treated. 

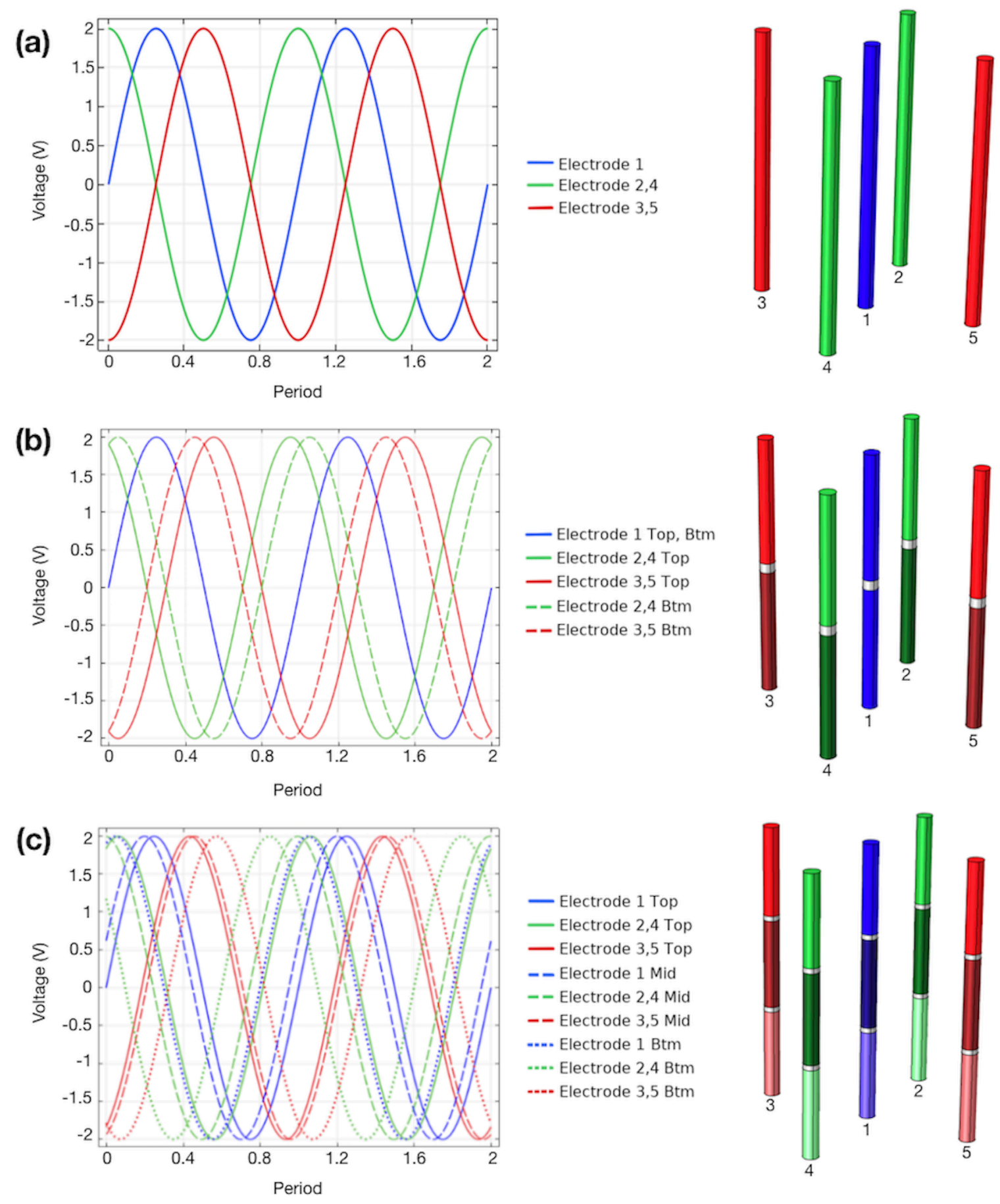

Fig. 5. Input voltage sine waves of optimal phase shift configurations with corresponding contact geometries for (a) single contact, (b) 2 contacts, and (c) 3 contacts. Two periods of the waveforms are shown. Contacts of the same colour have the same phase shift, contacts of different hue of the same colour differ slightly in phase shift and different colours differ maximally in phase shift. 



Fig. 6. Animations of $1 \mathrm{~V} / \mathrm{cm}$ isosurface coverage (red) of tumor volume (cyan) over time. Normalized electric field vectors show the field direction over time for both (a) single contact, 5 electrode and (b) 3contact, 5 electrode models. 
(a)



Electric Field (b)

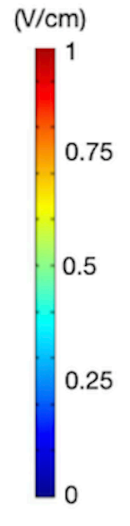

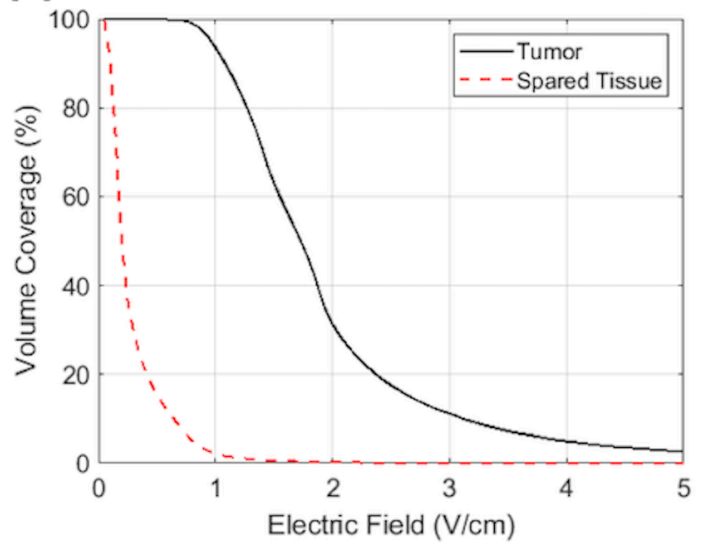

Fig. 7. (a) Average temporal electric field maps and corresponding animations of field coverage over time for a 5 electrode tissue sparing example with optimally placed and programmed electrodes. (b) Electric field volume histogram of the percent tumor volume and spared tissue receiving at least a certain temporal average electric field value $(\mathrm{V} / \mathrm{cm})$.
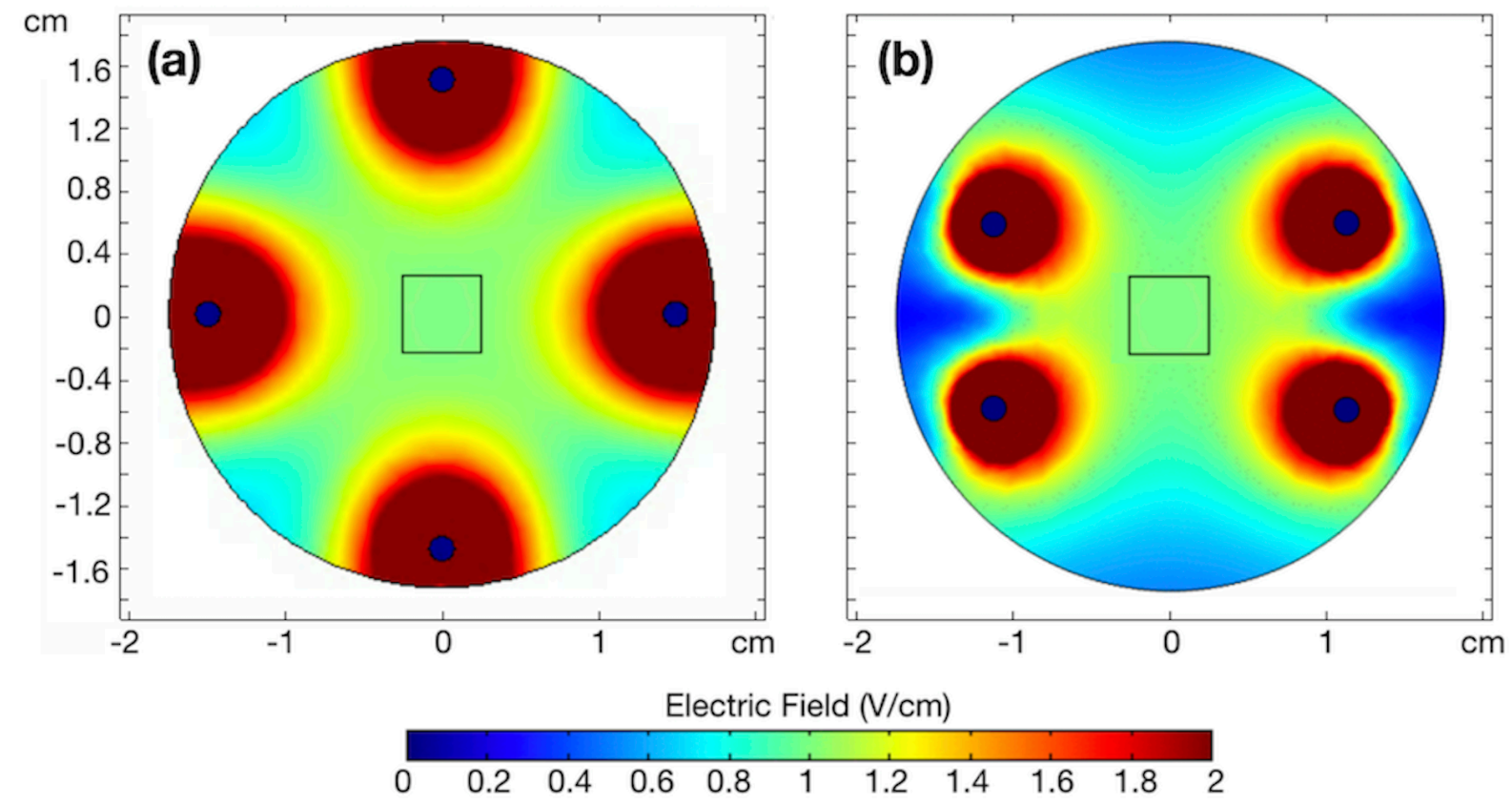

Fig. 8. Time average electric field magnitude $(\mathrm{V} / \mathrm{cm})$ for (a) rotating electric field and (b) non-rotating electric field. (Animation includes field vectors). 